GUPTA, C.R.; YADAV, R.D.S. Genetic variability and path analysis in chili (Capsicum annuum Linn.). Genetica Agraria, v. 38, p. 425 - 432, 1984.

MIRANDA, J.E.C. de; CRUZ, C.D.; PEREIRA, A.S. Análise de trilha e divergência genética de cultivares e clones de batata-doce. Revista Brasileira de Genética, Ribeirão Preto, v. 11, n. 4 , p. $881-892,1988$.
MODE, J.C.; ROBINSON, H.F. Pleiotropism and the genetic variance and covariance. Biometrics, v. 15, p. 518 - 537, 1959.

MONTGOMERY, D.C.; PECK, E.A. Introduction to linear regression analysis. New York: John Wiley \& Sons, 1981, 504 p.
SANTOS, C.A.F.; CAVALCANTI, J.; PAINI, J.N.; CRUZ, C.D. Correlações canônicas entre componentes primários e secundários da produção de grãos em guandu (Cajanus cajan (L.) MILLSP). Revista Ceres, Viçosa, v. 41, n. 236, p. 459 - 464, 1994.

VENCOVSKY, R.; BARRIGA, P. Genética biométrica no fitomelhoramento. Ribeirão Preto: Sociedade Brasileira de Genética, 1992, 496p.

SILVA, J.B.C.; NAKAGAWA, J. Metodologia para avaliação da resistência de péletes. Horticultura Brasileira, v. 16, n.2, p.118 -122, novembro 1998.

\title{
Metodologia para avaliação da resistência de péletes ${ }^{1}$.
}

\author{
João B.C. da Silva ${ }^{2}$; João Nakagawa ${ }^{3}$ \\ ${ }^{2}$ Embrapa - Hortaliças, C. Postal 218, 70.359-970 Brasília - DF \\ ${ }^{3}$ UNESP - Faculdade de Ciências Agronômicas, C. Postal 237, 18.603.970 Botucatu - SP.
}

\section{RESUMO}

A rigidez da camada de peletização depende da proporção de cimentante na sua constituição. Os péletes devem ser suficientemente rígidos para manterem a sua integridade física durante a classificação, no transporte, no manuseio ou na semeadura mecanizada. Entretanto, deve-se utilizar o mínimo de cimentante (adesivo), pois estes são geralmente produtos visguentos e a viscosidade da solução retida nos poros da camada de peletização, após a irrigação, afeta a drenagem da água e consequentemente a troca gasosa entre a semente e o ambiente externo ao pélete. Para avaliar a resistência física dos péletes, adaptou-se uma prensa confeccionando-se um anelde-prova com superfície interna de $10 \mathrm{~cm}^{2}$ e êmbolo de $8,7 \mathrm{~cm}^{2}$. Determinou-se a força necessária para reduzir cada milímetro da camada de pélete colocada no anel e, tomando-se o segmento retilíneo da curva originada das determinações, observou-se que a faixa de 5 a $20 \%$ de redução percentual do volume de péletes apresentou praticamente os mesmos valores de resistência para amostras de 20,30, 40 ou $50 \mathrm{ml}$ de péletes. Estabelecendo-se como índice de resistência, a força necessária para reduzir $10 \%$ do volume da amostra, partindo de uma amostra de $20 \mathrm{ml}$ e utilizando-se anel-de-prova de 10 $\mathrm{cm}^{2}$, mediu-se a resistência de quatorze tipos de péletes. Os péletes confeccionados com areia mais bentonita apresentaram os menores índices de resistência. Quando os péletes haviam recebido o acabamento com calcário mais cola à base de acetato de polivinila (PVA), apresentaram resistência intermediária e aqueles confeccionados com cola PVA como único cimentante, apresentaram os maiores índices.

Palavras-chave: pélete, semente, qualidade, resistência.
ABSTRACT

\section{Methods to evaluate pellet firmness.}

The amount of cement (adhesive) used for seed pelleting will determine its firmness. The pellets must be sufficiently firm to maintain their physical integrity during handling, processing, transportation and mechanical sowing. However, it is necessary to use the minimum of adhesive, because it inflicts viscosity to the water solution retained in the pores of the pellets after irrigation, affecting the water drainage and consequently, the gas exchange between seed and the environment outside the pellet. To measure the physical resistance of the pellets, a press with a gauge was adapted, making a test-ring with $10 \mathrm{~cm}^{2}$ of internal surface and 8.7 $\mathrm{cm}^{2}$ of piston. The force necessary to reduce each milimeter of the pellet sample layer closed inside the test-ring was determined. The retilineus segment of the curve made by these determinations corresponded to the range between $5 \%$ and $20 \%$ of the pellet volume reduction. In this range, the force necessary to reduce each percentage of volume was practically the same for samples of 20 , 30,40 or $50 \mathrm{ml}$ of pellet. A resistance index was proposed as the force necessary to reduce $10 \%$ of the sample volume, employing 20 $\mathrm{ml}$ of sample and the test-ring of $10 \mathrm{~cm}^{2}$. On the basis of this criteria, fourteen types of pellets were evaluated. Pellets made with sand and bentonite presented the smallest resistance index. The same pellets when covered with a fine lime finishing cover and glue of polivinil acetate (PVA) presented an intermediate value for resistance index and pellets made with lime and PVA glue were the most resistant.

Keywords: pellet, seed, coat, pellet-quality, firmness.

\section{(Aceito para publicação em 05 de outubro de 1998)}

$\mathrm{O}$ péletes não devem se desmanchar ou quebrar durante o processo de classificação, no transporte, no manuseio ou na semeadura mecanizada. Para evitar a sua desintegração, são utilizados os cimentantes (adesivos) que são aplicados por via úmida, em seguida secados e, após a semeadura, reidratados por meio de irrigação. A camada de peletização é relativamente delgada (menor que dois milímetros de espessura), mas é constituída por partículas finas, bem arranjadas e aderidas entre si, formando uma capa intimamente aderida à superfície da semente. Esta constituição implica em se ter o mínimo de porosidade e o máximo de força de retenção da água, dificultando a sua drenagem.

$\mathrm{O}$ arranjo das partículas finas e a ocupação dos espaços entre elas pelo

1 - Parte do trabalho para elaboração de tese de doutorado em Agronomia, área de concentração em Horticultura, pela Faculdade de Ciências Agronômicas - UNESP - Câmpus de Botucatu - SP. 
cimentante e pela água de irrigação, formam barreira à troca gasosa entre a semente e o ambiente externo ao pélete, diminuindo o suprimento de oxigênio, muito necessário na fase de germinação. Este é o principal fator restritivo à germinação das sementes peletizadas, segundo trabalhos realizados por Sachs et al. (1981), Mukhin et al. (1982), Ota (1982) e Sachs et al. (1982).

As partículas sólidas do componente de enchimento podem se agregar por força de coesão ou cargas eletrostáticas que atuam mais facilmente quando se adiciona água ao meio. Por isso, é possível fazer péletes sem aplicação de adesivos ou cimentantes. Porém, formamse estruturas de baixa resistência, que podem se desmanchar durante o processo de secagem ou no manuseio (Scott, 1989).

A firmeza da ligação entre as partículas define a consistência do pélete e, quanto maior a proporção do ingrediente cimentante, ou maior a superfície de contato, considerando tanto o tamanho das partículas quanto a sua porosidade, maior é a ação de fixação das estruturas.

Em razão da força de coesão entre as partículas e do arranjo a que são submetidas, à medida que vão se agregando à semente em camadas sucessivas, forma-se um núcleo compacto que não necessita ser fortemente fixado pelo cimentante (Scott, 1989).

A afinidade entre o cimentante e os demais ingredientes, ou o equivalente ao poder de fixação do produto é fator importante, no sentido de se utilizar o menor volume de solução ou a menor proporção de cimentante no pélete. Embora não se tenha encontrado qualquer bibliografia relacionada com este assunto, espera-se que, utilizando menor quantidade de cimentante, se tenha, ao reidratar os péletes após a semeadura, uma solução de baixa viscosidade na camada de peletização, o que deve facilitar a drenagem da água retida nos poros, favorecendo assim, a sua desobstrução e, conseqüentemente, a troca gasosa. Isto deve ocorrer porque os adesivos são, geralmente, produtos visguentos e a viscosidade da solução ou da suspensão depende da concentração.

A definição da proporção de cimentante implica portanto no dilema entre a necessidade de se ter resistência física, e a limitação de uso de cimentantes, sendo então necessário avaliar a resistência dos péletes, para que se possa utilizar a menor concentração possível de cimentantes, sem afetar a qualidade final do produto.

O objetivo deste trabalho foi adaptar uma prensa para avaliar a resistência dos péletes, por não se ter encontrado nenhuma descrição de metodologia para esta finalidade.

\section{MATERIAL E MÉTODOS}

Ensaio 1 - Adaptação da prensa Utilizou-se uma prensa dotada de anel dinamométrico com capacidade máxima de $200 \mathrm{kgf}$, medidor de espessura (centésimos de milímetro) da camada de deformação e macaco mecânico do tipo parafuso, acionado por manivela.

$\mathrm{Na}$ impossibilidade de se medir a resistência de cada pélete, optou-se por utilizar como índice de resistência, a força necessária para reduzir determinado percentual do volume de uma amostra de pélete, definindo-se também a superfície de ação da força. Para isso, amostras de volume definido foram colocadas dentro de um anel metálico (anel-de-prova) e sofreram deformações proporcionais ao seu volume, utilizando um êmbolo metálico ajustado ao diâmetro interno do anel-de-prova e pressionado pelo macaco mecânico.

Foram confeccionados dois conjuntos de anel-de-prova mais êmbolo, com diâmetro interno de $50 \mathrm{~mm}$ e $34 \mathrm{~mm}$, os quais corresponderam às superfícies de $20 \mathrm{~cm}^{2}$ e $8,7 \mathrm{~cm}^{2}$, respectivamente. Os conjuntos foram confeccionados com diferença mínima de diâmetro, suficiente para o encaixe entre as peças. Com isso, qualquer grânulo que se desprendia do pélete e atingia a região de contato entre o êmbolo e o anel-de-prova, causava grande atrito e resistência à movimentação das peças, sendo portanto grande fonte de erro.

Para evitar o inconveniente, foram confeccionados anéis de material plástico com o mesmo diâmetro do êmbolo, com espessura de aproximadamente 0,5 $\mathrm{mm}$ e de diversas alturas, permitindo escolher o anel plástico de acordo com o volume da amostra e a espessura da camada a ser comprimida, de forma que a espessura do anel fosse pelo menos o dobro da espessura da camada a ser compactada.

$\mathrm{O}$ anel de material plástico foi ajustado à região de contato entre as peças metálicas, impedindo a entrada de materiais sólidos naquele local. Desta forma, as partículas que entravam entre as superfícies dos anéis (de plástico e de metal) não causavam grande atrito em razão da plasticidade do material.

Utilizando-se dois tipos de pélete, sendo um confeccionado com areia e outro com calcário, ambos cimentados com cola à base de acetato de polivinila (PVA), mediu-se a força necessária para reduzir cada milímetro da altura da camada de pélete, avaliando-se amostras de 30 e $50 \mathrm{ml}$ de péletes.

Confeccionou-se outro conjunto de anel mais êmbolo, com superfície interna de $10 \mathrm{~m}^{2}$ (diâmetro de $35,7 \mathrm{~mm}$ ), para facilitar o cálculo da espessura da camada a ser reduzida (taxa de compressão) e se ter uma relação decimal entre força e a superfície de ação.

Utilizando o anel de $10 \mathrm{~cm}^{2}$ e o êmbolo de $8,7 \mathrm{~cm}^{2}$ verificou-se que se obtinham praticamente as mesmas leituras que as obtidas com o uso do anel plástico, pois os péletes em teste eram classificados por peneira e tinham diâmetro superiores a dois milímetros, enquanto que a diferença entre o diâmetro do anel e do êmbolo era de 2,3 mm. Tendo-se o cuidado de centralizar o êmbolo, o afastamento entre as peças era de aproximadamente $1 \mathrm{~mm}$ e portanto nenhum pélete deixava de receber a força de compressão e as partículas sólidas não tinham granulometria suficiente para causar atrito entre as peças metálicas, dispensando-se portanto o uso do anel de plástico.

Ensaio 2 - Avaliação dos péletes - Definida a possibilidade do uso do equipamento na mensuração da resistência de péletes, utilizou-se os aneis-deprova com 10 e $20 \mathrm{~cm}^{2}$ de superfície interna para fazer avaliação da força necessária para comprimir $5 ; 10 ; 15 ; 20 \mathrm{e}$ $25 \%$ do volume de amostras de 10; 20; 30; 40 e $50 \mathrm{ml}$ de pélete, utilizando-se péletes de semente de alface confeccionados com areia e com calcário dolomítico como ingredientes básicos e com 
cola à base de PVA como cimentante. Os péletes apresentavam grau de umidade de $0,45 \%$ e foram classificados em peneira, utilizando-se os que passaram pela peneira de 9,5 e foram retidos na de 7,5/64 de polegada $(3 \mathrm{~mm})$.

Foram feitas análises de correlação para cada dupla de seqüência de dados (entre tipos de péletes), visando escolher o melhor tamanho de amostra, o melhor anel-de-prova e a melhor taxa de compressão, bem como análise de regressão para a diferença da força medida para os dois tipos péletes, visando escolher o critério de avaliação mais estável. Fez-se também análise de variância para verificar a importância relativa de cada fator e variação das medições.

Ensaio 3 - Avaliação da resistência de vários tipos de péletes - Utilizou-se anel-de-prova com $10 \mathrm{~cm}^{2}$ e duas amostras de $20 \mathrm{ml}$ dos quatorze tipos de péletes de sementes alface com a seguinte codificação: $\mathrm{ACB}, \mathrm{ACBa}, \mathrm{ACP}$, AFBa, AFB, AGB, AGBa, AP, CFB, CFBa, CGB, CGBa, CP e SP, sendo: A - areia; B - bentonita (como cimentante); C - calcário; F - granulometria fina; $\mathrm{G}$ - granulometria grossa; $\mathrm{P}$ - cola à base de PVA; $\mathrm{S}$ - serragem de eucalipto; a - acabamento com calcário calcítico mais cola à base de PVA. Mediu-se a força necessária para o esmagamento até atingir as taxas de compressão correspondentes a $5 ; 10 ; 15 ; 20 \mathrm{e}$ $25 \%$ do volume das amostras (redução de 1 até $5 \mathrm{~mm}$ de altura da camada de amostra).

Os dados foram analisados segundo o modelo de experimento inteiramente casualizado, e as médias comparadas por contrastes.

Para permitir a comparação entre as taxas de compressão, calcularam-se as forças necessárias para comprimir cada $1 \%$ do volume das amostras (dividiu-se o valor da força pela taxa de compressão), fazendo-se então, a análise de variância, considerando a taxa de compressão como variável independente.

\section{RESULTADOS E DISCUSSÃO}

Ensaio 1 - Fez-se a plotagem dos dados em gráfico e verificou-se que em todos os casos, a força necessária para

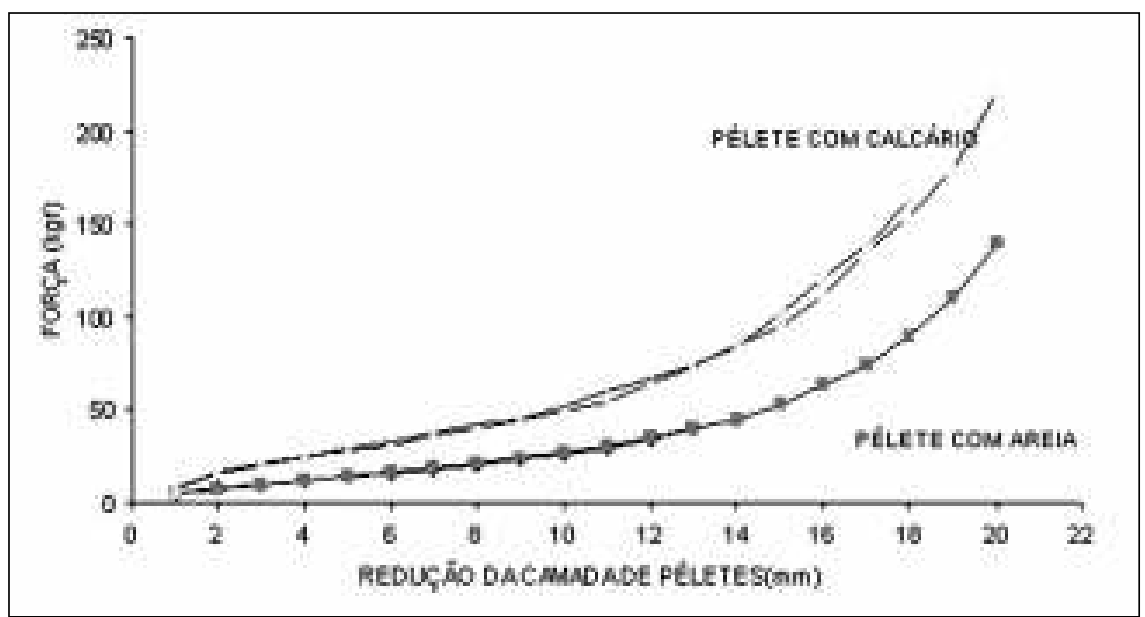

Figura 1. Força exigida para comprimir a camada correspondente a $50 \mathrm{ml}$ de péletes confeccionados com areia e com calcário, acondicionados em anel-de-prova de $10 \mathrm{~cm}^{2}$. Botucatu, UNESP, 1996.

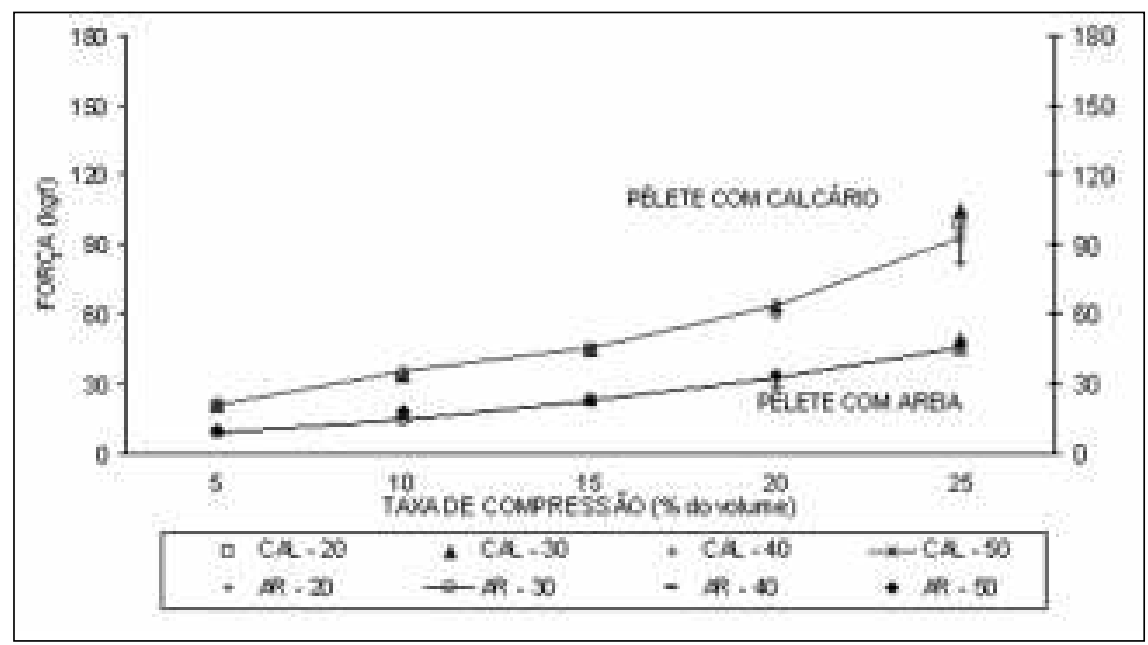

Figura 2 . Resistência de dois tipos péletes ao esmagamento, utilizando o anel-de-prova com $10 \mathrm{~cm}^{2}$, volumes de amostra de 20; 30; 40 e $50 \mathrm{ml}$ e taxas de compressão de $5 \%$ a $25 \%$ do volume $(\mathrm{CAL}=$ pélete com calcário, $\mathrm{AR}=$ pélete com areia). Botucatu, UNESP, 1996.

comprimir os primeiros milímetros da camada era semelhante para ambas amostras e que, ao comprimir excessivamente as amostras, os valores de força eram tão elevados que correspondiam provavelmente à força de compressão da massa de materiais e não mais dos péletes em si (Figura 1). Verificou-se ainda que havia, também em todos os casos, uma faixa de medições em que os dados seguiam tendência retilínea e próximo do paralelismo entre si (entre tipos de pélete e entre volume de amostra), indicando ser a faixa de compressão de 5 a $10 \mathrm{~mm}$, a que oferecia a maior estabilidade das medições.

Calculando-se o volume percentual correspondente à altura da camada reduzida pela compressão, verificou-se que a faixa de comportamento retilíneo dos dados estava entre 5 e $25 \%$ do volume das amostras. Os dados correspondentes à esta faixa de leitura foram submetidos às análises de correlação e de regressão e obteve-se índices de correlação superiores a 99\% quando se compararam valores para o mesmo tipo de pélete e correlações de $92,4 \%$ e $94,6 \%$ quando se compararam valores entre os tipos de pélete, para os anéis-de-prova de 20 e de $8,7 \mathrm{~cm}^{2}$, respectivamente. Nas análises de regressão obtiveram-se valores de índices de ajuste $\left(\mathrm{R}^{2}\right)$ superiores a $98 \%$ para os modelos retilíneos ajustados a cada seqüência de medição.

A média das medições foi de 36,9 kgf, com mínimo de 8 kgf e máximo de 54 kgf. A diferença entre os índices de 
resistência para os dois péletes em teste foi de 14,2 kgf quando se utilizou amostra de $30 \mathrm{ml}$, taxa de compactação de 5\% e anel de $8,7 \mathrm{~cm}^{2}$ e de $35,3 \mathrm{kgf}$ quando se utilizou amostra de $50 \mathrm{ml}$, taxa de compactação de $25 \%$ e anel de $20 \mathrm{~cm}^{2}$. Deste teste concluiu-se que o equipamento era útil para a avaliação da resistência mecânica ao esmagamento dos péletes e que a faixa mais apropriada para as determinações estava entre 5 e $25 \%$ de taxa de compressão da amostra. Concluiu-se ainda que, quanto maior o volume da amostra, a taxa de compressão e a superfície interna do anel, maior era a faixa de diferenciação dos índices de resistência (distanciamento entre índices de resistência de péletes distintos).

Ensaio 2 - Analisando-se os dados de resistência ao esmagamento de amostras com volumes de 20, 30, 40 e $50 \mathrm{ml}$, de dois tipos de péletes, verificou-se que a força exigida para comprimir determinada porcentagem do volume da amostra não dependeu do volume, quando se utilizou o anel-de-prova com 10 $\mathrm{cm}^{2}$ de superfície interna (Figura 2), resultando em curvas praticamente sobrepostas, para ambos os tipos de péletes e também a mesma proporcionalidade dos resultados entre os tipos.

Quando se utilizou o anel-de-prova com $20 \mathrm{~cm}^{2}$, obtiveram-se curvas diferentes, mas próximas do paralelismo entre si (Figura 3), principalmente se consideradas para cada tipo de pélete, indicando a mesma tendência dos resultados obtidos com o outro anel. Notouse também este resultado na análise de correlação, que apresentou índices entre 95 e $99 \%$ quando se comparou as seqüências de dados para cada tipo de pélete, e índices de 78 a 99\% quando se analisou as seqüências de dados relativas à diferença de valores entre os dois tipos de péletes.

$\mathrm{Na}$ análise de regressão por "stepwise" verificou-se que todos os fatores (superfície do anel, volume da amostra e taxa de compressão), interferiram significativamente nos resultados, mas que a taxa de compressão foi o componente mais influente do modelo, sendo responsável por $49 \%$ da variação dos resultados, seguido pelo fator superfície do anel, com $37 \%$ dos efeitos, e do fator volume, com $5 \%$, totalizando $91 \%$, que foi o coeficiente do modelo completo.

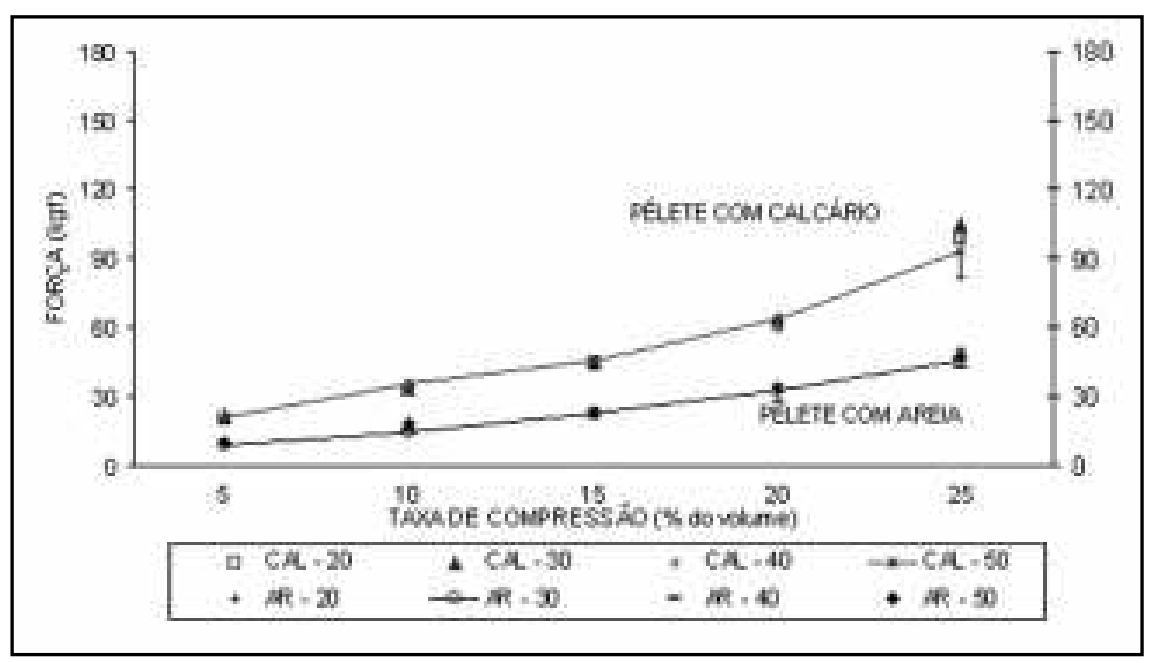

Figura 3 . Resistência de dois tipos péletes ao esmagamento, utilizando o anel-de-prova com $20 \mathrm{~cm}^{2}$, volumes de amostra de 20; 30; 40 e $50 \mathrm{ml}$ e taxas de compressão de 5 a $25 \%$ do volume $(\mathrm{CAL}=$ pélete com calcário, $\mathrm{AR}=$ pélete com areia). Botucatu, UNESP, 1996.

Ao trabalhar com taxas de compressão e volume reduzidos, obteve-se menor diferença entre os tipos de péletes e portanto, menor poder discriminatório das medições. Considerando que o método causa a destruição da amostra e que os resultados obtidos com o anel de 10 $\mathrm{cm}^{2}$ foram estáveis e suficientes para discriminar os tipos de péletes, propõese utilizá-lo neste tipo de determinação.

Utilizando-se a taxa de compressão de $5 \%$ verificou-se que os valores obtidos se aproximavam entre si e, com estes valores, os péletes foram agrupados de forma diferente do obtido com a média das determinações feitas com as diversas taxas de compressão (Tabela 1). Este parâmetro foi também o que apresentou os menores índices de correlação (92 a 97\%) com as demais determinações.

Ao comprimir $25 \%$ do volume das amostras, obteve-se maior desvio dos resultados, indicando que parte da força aplicada poderia estar sendo absorvida por outro motivo, que não o do esmagamento dos péletes, concluindo-se que as taxas de compressão de 5 e de $25 \%$ foram impróprias para a determinação da resistência.

As forças obtidas com as taxas de compressão de $10 ; 15$ e $20 \%$ foram as que apresentaram os maiores índices de correlação (cerca de 99\%) entre si e com a média das determinações, agrupando de forma muito semelhante os quatorze tipos de péletes, para cada taxa de compressão considerada.

Os valores obtidos com a taxa de compressão de 20 e $25 \%$ são também altamente correlacionados entre si e com a média, mas apresentaram quase o dobro do coeficiente de variação obtido com as taxas de 10 e $15 \%$.

Utilizando como índice de resistência a força necessária para comprimir cada $1 \%$ do volume da amostra (dividindo o valor da força pela taxa de compressão) foi possível comparar estatisticamente os valores obtidos nas diversas taxas de compressão e também com a média, uma vez que se uniformizou o dimensionamento do fator estudado.

Verificou-se que este índice foi mais alto quando se utilizaram as taxas de compressão de 5 e de $25 \%$, indicando que nestes casos mediam-se forças diferentes das necessárias para romper as estruturas dos péletes. O menor valor médio deste índice foi obtido com a taxa de compressão de $15 \%(2,66 \mathrm{kgf} / 1 \%$ de compactação), sendo que este não diferiu dos valores obtidos com a taxa de $10 \%$ (2,65 kgf/ 1\% de compactação), que por sua vez não diferiu da de $20 \%$ (2,70 kgf / 1\% de compactação), donde se concluiu ser esta a faixa mais estável de medições.

Utilizando como termos de comparação, tanto a diferença mínima significativa (DMS) para a comparação das médias, quanto o valor de "F", que é a relação entre a variância devida aos tratamentos e a variância devida ao erro experimental, obteve-se, na avaliação de resistência em que se utilizou a taxa de compressão de $15 \%$, o maior valor de "F" e menor valor de DMS, indicando que aquele critério de avaliação era o 
Tabela 1. Força necessária para reduzir de 5 a $25 \%$ do volume de $20 \mathrm{ml}$ de amostras de péletes de sementes de alface, utilizando o anel-deprova com $10 \mathrm{~cm}^{2}$ de superfície interna. Botucatu, UNESP, 1996.

\begin{tabular}{|c|c|c|c|c|c|c|c|c|c|c|c|c|}
\hline \multirow{2}{*}{ Pélete $^{1}$} & \multicolumn{12}{|c|}{ taxa de compressão ${ }^{2}(\%)$} \\
\hline & \multicolumn{2}{|r|}{5} & \multicolumn{2}{|c|}{10} & \multicolumn{2}{|c|}{15} & \multicolumn{2}{|c|}{20} & \multicolumn{2}{|c|}{25} & \multicolumn{2}{|c|}{ média } \\
\hline \multicolumn{13}{|c|}{ força (kgf) } \\
\hline $\mathrm{CP}$ & $45,8 \mathrm{a}$ & & $85,5 \mathrm{a}$ & & $122,3 \mathrm{a}$ & & $174,7 \mathrm{a}$ & & $231,9 a$ & & $132,04 \mathrm{a}$ & \\
\hline $\mathrm{ACP}$ & $23,5 \mathrm{~b}$ & $b$ & $36,1 \quad 1$ & $\mathrm{bc}$ & 48,2 & $\mathrm{c}$ & 70,5 & $\mathrm{c}$ & 108,4 & $\mathrm{c}$ & 57,34 & $\mathrm{c}$ \\
\hline CFBa & $21,1 \quad b$ & $b c$ & 34,9 & $\mathrm{bc}$ & 48,2 & $\mathrm{c}$ & 62,0 & $\mathrm{~cd}$ & 86,1 & $\mathrm{~cd}$ & 50,48 & $\mathrm{~d}$ \\
\hline CGBa & 19,3 & $\mathrm{c}$ & 33,7 & $\mathrm{c}$ & 47,6 & $\mathrm{c}$ & 60,2 & cde & 81,9 & cde & 48,55 & $\mathrm{~d}$ \\
\hline $\mathrm{ACBa}$ & 18,7 & $\mathrm{~cd}$ & 31,9 & $\mathrm{c}$ & 45,8 & $\mathrm{c}$ & 60,2 & cde & 82,5 & cde & 47,83 & $\mathrm{~d}$ \\
\hline SP & 15,7 & de & 40,4 & b & 68,1 & b & 101,2 & b & 143,9 & b & 73,85 & b \\
\hline AGBa & 13,9 & ef & 24,1 & $d$ & 33,7 & d & 45,8 & def & 61,4 & def & 35,78 & e \\
\hline CGB & 10,8 & fg & 18,7 & de & 28,9 & de & 42,8 & ef & 69,9 & def & 34,22 & e \\
\hline AFBa & 10,8 & $\mathrm{fg}$ & 18,7 & de & 24,7 & e & 34,9 & $\mathrm{f}$ & 44,0 & $\mathrm{f}$ & 26,63 & $\mathrm{f}$ \\
\hline CFB & 10,8 & $\mathrm{fg}$ & 17,5 & $\mathrm{e}$ & 24,7 & e & 39,8 & $\mathrm{f}$ & 62,6 & def & 31,08 & ef \\
\hline AP & 9,0 & $\mathrm{fg}$ & 15,1 & $\mathrm{e}$ & 22,3 & e & 34,9 & $\mathrm{f}$ & 56,0 & ef & 27,47 & $\mathrm{f}$ \\
\hline $\mathrm{ACB}$ & 4,2 & $\mathrm{~h}$ & 6,0 & $\mathrm{f}$ & 9,6 & f & 12,1 & $\mathrm{~g}$ & 19,3 & $\mathrm{~g}$ & 10,24 & \\
\hline AFB & 3,6 & $\mathrm{~h}$ & 4,8 & $\mathrm{f}$ & 6,0 & $f$ & 9,0 & $\mathrm{~g}$ & 15,1 & $\mathrm{~g}$ & 7,71 & \\
\hline AGB & 3,0 & $\mathrm{~h}$ & 4,2 & $\mathrm{f}$ & 6,6 & $\mathrm{f}$ & 9,0 & $\mathrm{~g}$ & 16,9 & $\mathrm{~g}$ & 7,95 & \\
\hline Média & $15,01 \mathrm{E}$ & & $26,55 \mathrm{I}$ & & 38,34 & & 54,08 & & 77,15 & & & \\
\hline C.V.(\%) & 5,0 & & 5,6 & & 4,6 & & 8,7 & & 8,8 & & 9,14 & \\
\hline
\end{tabular}

1 - Código dos materiais utilizados na confecção dos péletes: A - areia; B - bentonita (como cimentante); C - calcário; F - granulometria fina; G - gran. grossa; P - cola à base de PVA; S - serragem de eucalipto; a - acabamento com calcário calcítico mais cola à base de PVA.

2 - Porcentagem de redução do volume da amostra.

Os valores seguidos pela mesma letra, maiúscula na linha e minúscula na coluna, não diferem entre si pelo teste de Tukey, a 5\%.

que melhor discriminava ou agrupava os péletes quanto ao fator avaliado.

Considerando que houve correlação muito estreita (99\%) entre as determinações feitas com as taxas de compressão de 10,15 e $20 \%$, e que o trabalho para fazer as três leituras é praticamente o mesmo de se fazer apenas uma, propõe-se fazer as três leituras, calcular o índice citado anteriormente, e obter a média das determinações. Para evitar trabalhar com números de baixo valor, deve-se multiplicar a média por dez, obtendo-se então o índice médio de resistência, definido como sendo a força necessária para reduzir $10 \%$ do volume da amostra. Esses cálculos podem ser resumidos na seguinte fórmula:

$$
\mathrm{Ir}_{\mathrm{m}}=\frac{\frac{\mathrm{F}_{10}}{10}+\frac{\mathrm{F}_{15}}{15}+\frac{\mathrm{F}_{20}}{20}}{3} \times 10 \text { sendo : }
$$

$\mathrm{Ir}_{\mathrm{m}}$ - Índice médio de resistência,

$\mathrm{F}_{10}, \mathrm{~F}_{15}$ e $\mathrm{F}_{20}$ - Força necessária para reduzir em 10,15 e $20 \%$ do volume da amostra, respectivamente.
Ensaio 3 - Utilizando como parâmetro o índice de resistência citado anteriormente, verificou-se que os péletes confeccionados com calcário e cola à base de PVA foram os mais resistentes ao esmagamento e que os péletes confeccionados com areia, utilizando bentonita como cimentante e sem aplicação da camada de acabamento, foram os de menor resistência.

Fazendo-se agrupamentos dos péletes, concluiu-se que o calcário formou péletes mais resistentes que a areia; que a cola à base de PVA atuou como cimentante mais forte que a bentonita; que aplicação da camada de acabamento com cola à base de PVA mais calcário calcítico aumentou a firmeza dos péletes; e que a mistura de areia mais calcário formou péletes com resistência intermediária.

Diversos tipos de péletes foram avaliados com esta metodologia, e os resultados fazem parte de outro trabalho.

A adaptação da prensa permitiu avaliar a resistência dos péletes ao esmagamento, obtendo-se o índice de resistência, que pode auxiliar na definição dos padrões de qualidade e na determinação das proporções mais adequadas de ingredientes.

\section{LITERATURA CITADA}

MUKHIN, V.D.; MEDVEDEV, V.G.; SARBASH, A.K. Productivity of parsley and carrot after treating the seeds in water with oxygen and pelleting. Horticulutral Abstracts, v. 52, p. 87, 1982. (abs. 891)

OTA, Y. Promotion of emergence and establishment of rice seedlings by using calcium peroxidecoated seeds in direct sowing on flooded paddy fields. Japan Agriculture Reserch Quartery, v. 15, n. 4, p. 221- 6, 1982.

SACHS, M.; CANTLIFFE, D.J.; NELL, T.A. Germination studies of clay-coated sweet pepper seeds. Jouranl of the American Society for Horticultural Science, v. 106, p. $385-9,1981$.

SACHS, M.; CANTLIFFE, D.J.; NELL, T.A. Germination behavior of sand-coated sweet pepper seed. Journal of the American Society for Horticultural Science, v. 107, p. 412 - 6, 1982.

SCOTT, J.M. Seed coatings and treatments and their effects on plant establishment. Advances in Agronomy, v. 42, p. 43 - 83, 1989. 